\title{
Effect of High Levels of Human Chorionic Gonadotropin and Estradiol on Degree of Hyperemesis Gravidarum
}

\begin{abstract}
* Corresponding Author:
Ahmed G. A. A. Shaheen

aga12121989@yahoo.com

Received for publication June 21, 2021; Accepted July 23, 2021; Published online July 23, 2021.

Copyright The Authors published by Al-Azhar University, Faculty of Medicine, Cairo, Egypt. Users have the right to read, download, copy, distribute, print, search, or link to the full texts of articles under the following conditions: Creative Commons Attribution-Share Alike 4.0 International Public License (CC $B Y-S A$ 4.0).

doi: 10.21608 /aimj.2021.81643.1508

${ }^{I}$ Resident of Obstetrics \& Gynecology, M.B.B.CH, Faculty of Medicine, El Fayoum University, Egypt.

${ }^{2}$ Obstetrics and Gynecology Department, Faculty of Medicine, Al-Azhar University Cairo, Egypt.
\end{abstract}

Ahmed G. A. A. Shaheen ${ }^{1, *}$ M.B.B.Ch, Yehia A. Wafa ${ }^{2}$ MD, Fahd A. El-Omda ${ }^{2}$ MD

\begin{abstract}
Background: Hyperemesis gravidarum is a condition that affects pregnant women (HG),serum levels of human chorionic gonadotropin (hCG) and estradiol are increased than in pregnant women who are not influenced.

Aim of the work: To learn more about how the elevation of human chorionic gonadotropin levels, and estradiol on the intensity of hyper emesis gravidarum during the first trimester.

Patients and methods: At El Hussein University Hospital, our prospective study was conducted from May 2020 to Nov. 2020; duration of the study was 7 months. The key sign of severe Hyper Emesis Gravidarum was a lengthy stay in the hospital (HG).The term "extended hospital stay" described as stay $\geq 4$ days, as this cut-off value demarcates the top quartilef orhospitalization timein our study population.

Results: There was a highly significant difference between cases with hospital stay $<4$ days and cases with hospital stay $\geq 4$ days in HCG cases. cases with longer stay have higher median HCG (300637) compared to cases with $<4$ days stay (35029.5). However, no significant that showed difference was found between cases with hospital stay< 4 days and cases with hospital stay $\geq 4$ days was present as regard estradiol.

Conclusion: Hyperemesis gravidarum is a problem that involves severe nausea, vomiting, and anorexia during pregnancythat causes dehydration and weight loss in early pregnancy. Study found that there is a significant correlation between HCG and hyperemesis gravidarum and there is no significant correlation between estradiol and hyperemesis gravidarum andthere is a strong link between presence of acetone andhyperemesis gravidarum intensity.So, The higher the levels of HCG the more the intensity of hyperemesis gravidarum ; ( i.e high levels of HCG increase the severity of $\mathrm{HG}$ ).

Keywords: Hyperemesis gravidarum, human chorionic gonadotrophine, Estradiol.
\end{abstract}

Disclosure: The authors have no financial interest to declare in relation to the content of this article. The Article Processing Charge was paid for by the authors. Authorship: All authors have a substantial contribution to the article.

\section{INTRODUCTION}

Emesis gravidarum, or pregnancy nausea and vomiting, is a multifactorial disorder that has major negative impact on the mother's and fetus's health and wellbeing. It is important to recognize, investigate, and treat this illnes ${ }^{1}$.

Symptoms of morning sickness, which are incorrectly called morning sickness, usually begin between the first and second missing menstrual period and last for about 14 to 16 weeks. Although nausea and vomiting are usually worse first thing in the morning, they can last all day ${ }^{2}$.

In $0.3 \%$ to $3 \%$ of pregnancies, hyperemesis gravidarum (HG) develops. HG is the second leading cause forwomen having live deliveries to be admitted to the hospital throughout their pregnancy. ${ }^{3}$
Female fetus sex, socioeconomic conditions, and ethnicity all play a role in hyper emesis gravidarum. HG is commonly distinguished from the more recurrent vomiting and nausea thatdevelops during

pregnancy, that impacts $80 \%$ of all pregnant women . and necessitates hospitalization. ${ }^{4}$.

HG's cause has yet to be determined. It appears to be multifactorial in nature, and it could be the result of a number of unconnected factors. Endocrine factors such as high human chorionic gonadotrophine, estradiol, and steroid hormone levels, gastrointestinal tract dysfunction, psychological causes, anatomical variations, genetic incompatibility, immunological factors, vitamin B6 deficiency, and trace-element deficiency are all thought to play a role in $\mathrm{HG}^{5}$.

Women who were unable to manage severe nausea and vomiting symptoms and chose abortion have been reported in case reports. Furthermore, over half of working pregnant women said that their job efficiency has been harmed by nausea and vomiting, 
and around a quarter of these women have had to take time off work due to these problems ${ }^{6}$.

This is making it also socioeconomic problem. The reported estimated cost for hospital care alone is more 500 million $\$$ for the 59.000 women hospitalized with hyper emesis gravidarum in the United State annually ${ }^{7}$.

In women with HG, anelevated HCG level is linked to more severe vomiting and a delay in the clearance of aberrant biochemical markers. In HG, the level of maternal serum oestrogen is higher, but not in nausea and vomiting during pregnancy.

\section{PATIENTS AND METHODS}

A prospective study was done at El Hussein University Hospital from May 2020 to Nov. 2020 duration of the study was 7 months. An extended hospitalization was used asa main evidence of intensityfor Hyper Emesis Gravidarum (HG). . An extended hospital stay wasdescribed as a stay $\geq 4$ days, as this cut-off value demarcates the top quartilef or length of hospitalization period in the study population.

This study includes 80 pregnant women during the first trimester of their ongoing pregnancy with HG. Hyper emesis gravidarumis usually described as persistent vomiting in pregnancy that interferes with fluid intake $\&$ nutrition. The maximum and minimum demarcations for HCG and estradiol were defined by using the power trend function in Microsoft office excel 2010 to graph a curve of right fit for the scatter grammes of HCG or estradiol levels vs. ultrasoundderived gestational age, as the power function produced the best fit curve depending on the $\mathrm{r} 2$ value. All points below the curve were designated "low," while those above it were marked "high." The providers were not informed about the HCG and estradiol levels.

On admission to the gynaecology ward, women with a suspected diagnosis of Hyper Emesis Gravidarum (HG) were enrolled. Following that, the patient's agreement was obtained after a brief explanation. HCG and estradiol levels were measured in venous blood as soon as feasible after admission, and the results were transferred to the hospital laboratory for analysis.

The Abbott Axsym Total HCG test and the Abbott Axsym Estradiol-6 assay were used to measure the levels of HCG and estradiol in the study participants. Using dilutional Techniques, the Abbott Axsym Total HCG Assay assesses HCG levels up to 1000 $\mathrm{mIU} / \mathrm{mL}$ (IU/L) and up to $1000000 \mathrm{mIU} / \mathrm{ml}$ using Dilutional Techniques, with a minimum detectable value of $2.0 \mathrm{mIU} / \mathrm{mL}$ (IU/L) as the least detectable concentration. A level of $10 \mathrm{pg} / \mathrm{mL}(36.7 \mathrm{pmol} / \mathrm{L})$ is required for the Abbott Axsym Estradiol-6 assay to be sensitive, and it can detect estradiol quantities up to $1000 \mathrm{pg} / \mathrm{mL}(3670 \mathrm{pmol} / \mathrm{L})$. When the serum samples contained quantities that exceeded the upper limits of the above-mentioned ranges, the laboratory made the necessary dilutions.

Inclusion criteria: Pregnantin first trimester less than 14 Week as determined by ultrasound, medicallyfree by history taking and by physical examination, vomiting more than 3 times a day and causes dehydration and loss of weight more than $3 \mathrm{~kg}$ or $5 \%$ of body weight, singleton uncomplicated pregnancies and first hospitalization.

Exclusion criteria: Pregnant in 2nd and 3rd trimester, medical disorders especially that cause vomiting as cholecystitis, appendicitis and colitis, multiple pregnancies and non-viable pregnancy.

The following criteria were applied to all cases: Getting a complete history with stressing on age, parity and gestational age, clinical examination including body weight, abdominal examination to exclude other causes of vomiting, trans-abdominal or transvaginal pelvic sonogram to confirm gestational age, to exclude multiple pregnancies and gestational trophoblastic disease. Estradiol and HCG values were measured in the venous blood quickly following admission, andwere given to our hospital's lab for analysis. Before beginning substantive treatment, a full blood count, ketones in urine (by dipstick), creatinine, urea, s.electrolytes, SGOT, SGPT and Bilirubin (total and direct) were all performed.

We decided to utilize ultrasound-derived gestational age and limit our trial to women who were 14 weeks pregnant, as HG usually manifests at this point and HCG levels peak late in the first trimester.

Questionnaire for patients included: name, age, parity, weight, gestational age, onset, duration of symptoms of H.G, hemoglobin (HB) level, acetone in urine.

In pluses, comments on urine analysis, liver enzymes, serum electrolytes, these data were obtained from patient sheets as all cases were admitted to the inpatient department.

Statistical method:The statistical analysis was carried out with the help of the SPSS v.15 program (SPSS Inc., Chicago, IL, USA). It was decided whether to compare means or ordinal variables using the Student t test, and the Mann-Whitney U test was employed for both. When dealing with categorical data, the Fisher exact test ( $2=2$ datasets) and the $\times 2$ test (for datasets greater than $2=2$ datasets) were utilized. For the purposes of adjusting for participant characteristics, laboratory data, and treatment mode, a multivariable logistic regression analysis (considering all factors with a crude p-0.2) was performed. A statistically significant result was defined as one with an adjusted p-value of less than 0.05 . All tests use two-sided. 


\section{RESULTS}

The table shows that the mean age, weight and GA among study cases was $26.9 \pm 6.1$ years, $67.2 \pm 6.8 \mathrm{Kg}$ and $10.8 \pm 5.2$ Weeks respectively. The median parity among cases was 2 children. Table1.

\begin{tabular}{|l|r|r|r|r|r|r|r|}
\hline & Mean & \pm SD & Minimum & Maximum & Median & \multicolumn{2}{|c|}{ IQR* } \\
\hline Age & 26.9 & 6.1 & 16.0 & 45.0 & 26.0 & 22.0 & 30.0 \\
\hline Weight & 67.2 & 6.8 & 55.0 & 85.0 & 67.5 & 62.0 & 71.0 \\
\hline $\begin{array}{l}\text { Gestational } \\
\text { Parity }\end{array}$ & 10.8 & 5.2 & 7.0 & 14.0 & 10.0 & 8.0 & 12.0 \\
\hline
\end{tabular}

*interquartile range

Table 1: Description of personal and medical data among cases

The table shows that the mean HCG was $197434.8 \pm 143817.9$ with a median of 256127. HCG was high among $55 \%$ of cases. The mean estradiol was $2141 \pm 946.5$ with a median of 2200 . Estradiol was high among $42.5 \%$ of cases. Table2.

\begin{tabular}{|c|c|c|c|c|c|c|c|c|}
\hline \multirow{2}{*}{ HCG } & & \multirow{2}{*}{$\begin{array}{c}\text { Mean } \\
197434.8\end{array}$} & \multirow{2}{*}{$\begin{array}{c}\text { 土SD } \\
143817.9\end{array}$} & \multirow{2}{*}{$\begin{array}{r}\text { Minimum } \\
8153.0\end{array}$} & \multirow{2}{*}{$\begin{array}{r}\text { Maximum } \\
403901.0\end{array}$} & \multirow{2}{*}{$\begin{array}{r}\text { Median } \\
256127\end{array}$} & \multicolumn{2}{|c|}{ IQR } \\
\hline & & & & & & & 31900 & 313191 \\
\hline \multicolumn{2}{|l|}{ Estradiol } & 2141 & 946.5 & 388.0 & 4913.0 & 2200.5 & 1291.0 & 2800.0 \\
\hline \multirow[t]{2}{*}{ HCG } & Normal & 36 & $45 \%$ & & & & & \\
\hline & High & 44 & $55 \%$ & & & & & \\
\hline \multirow[t]{2}{*}{ Estradiol } & Normal & 46 & $57.5 \%$ & & & & & \\
\hline & High & 34 & $42.5 \%$ & & & & & \\
\hline
\end{tabular}

Table 2: Description of HCG and estradiol level among cases

More than half of cases $(51.25 \%)$ were admitted in hospital for 4 or more days which mean sever hyper emesis gravidarum. Table 3

\begin{tabular}{|c|c|c|c|}
\hline \multicolumn{2}{|c|}{} & $\mathbf{N}$ & $\%$ \\
\hline \multirow{2}{*}{ Hospital stay } & $<4$ days & 39 & $48.75 \%$ \\
\cline { 2 - 4 } & $\geq 4$ days & 41 & $51.25 \%$ \\
\hline
\end{tabular}

Table 3: Descriptions of duration of hospital stay among cases.

There was no significant correlation between personal and medical data on one hand (age, parity, GA, weight and $\mathrm{Hb}$ ) and HCG level on the other hand.Table4

\begin{tabular}{|l|c|c|}
\hline \multirow{5}{*}{ Age } & & HCG \\
\hline \multirow{5}{*}{ Parity } & Rho* & -.051 \\
\cline { 2 - 3 } & $\mathrm{P}$ & .684 \\
\cline { 2 - 3 } & Sig & NS \\
\hline Gestational age & Rho* & -.099 \\
\cline { 2 - 3 } & $\mathrm{P}$ & .431 \\
\cline { 2 - 3 } & Sig & NS \\
\hline Weight & Rho* & .032 \\
\cline { 2 - 3 } & $\mathrm{P}$ & .801 \\
\cline { 2 - 3 } & Sig & NS \\
\hline & Rho* & -.015 \\
\cline { 2 - 3 } & P & .904 \\
\cline { 2 - 3 } & Sig & NS \\
\hline
\end{tabular}




\begin{tabular}{|l|c|c|}
\hline HB & Rho* & -.065 \\
\cline { 2 - 3 } & P & .604 \\
\cline { 2 - 3 } & Sig & NS \\
\hline
\end{tabular}

Table 4: Correlation between personal data and HCG leve

There was no significant correlation between personal and medical data on one hand (age, parity, Weight and $\mathrm{Hb}$ ) and estradiol level on the other hand, with exception of GA where it showed a positive significant correlation with estrsdiol level.Table 5

*spearmen correlation

\begin{tabular}{|c|c|c|}
\hline & & Estradiol \\
\hline \multirow[t]{3}{*}{ Age } & Rho* & -.216 \\
\hline & $P$ & .082 \\
\hline & Sig & NS \\
\hline \multirow[t]{3}{*}{ Parity } & Rho* & -.168 \\
\hline & $\mathrm{P}$ & .178 \\
\hline & Sig & NS \\
\hline \multirow[t]{3}{*}{ Gestational age } & Rho* & .442 \\
\hline & $P$ & .0001 \\
\hline & Sig & HS \\
\hline \multirow[t]{3}{*}{ Weight } & Rho* & -.127 \\
\hline & $P$ & .311 \\
\hline & Sig & NS \\
\hline \multirow[t]{3}{*}{ HB } & Rho* & .185 \\
\hline & $P$ & .137 \\
\hline & Sig & NS \\
\hline
\end{tabular}

Table 6: Correlation between HCG and estradiol level

There was no significant correlation between HCG and estradiol level this result againstthe scientific background.Table 6

\begin{tabular}{|l|l|r|}
\hline \multirow{4}{*}{ HCG } & & \multicolumn{1}{|c|}{ Estradiol } \\
& Rho* & .110 \\
\cline { 2 - 3 } & P & .380 \\
\cline { 2 - 3 } & Sig & NS \\
\hline
\end{tabular}

Table 6: Correlation between HCG and estradiol level

There was a highly significant difference between cases with hospital stay $<4$ days and cases with hospital stay $\geq 4$ days as regard HCG, cases with longer stay have higher median HCG (300637) compared to cases with <4 days stay (35029.5). However, no significant difference between cases with hospital stay< 4 days and cases with hospital stay $\geq 4$ days was present as regard estradiol.Table 7

\begin{tabular}{|c|c|c|c|c|c|c|c|c|c|}
\hline & \multicolumn{6}{|c|}{ Hospital stay } & \multirow[t]{3}{*}{ Statistic } & \multirow[t]{3}{*}{$\mathbf{P}$} & \multirow[t]{3}{*}{ Sig } \\
\hline & \multicolumn{3}{|c|}{$<4$ days } & \multicolumn{3}{|c|}{$\geq 4$ days } & & & \\
\hline & Median & \multicolumn{2}{|c|}{$\mathrm{IQR} *$} & Median & \multicolumn{2}{|c|}{ IQR* } & & & \\
\hline HCG & 35029.6 & 23900.0 & 118461.0 & 300637.1 & 275665.5 & 361065.0 & $5.2 * *$ & .001 & $\mathrm{HS}$ \\
\hline Estradiol & 1951.70 & 1059.60 & 2695.00 & 2552.60 & 1961.00 & 2925.00 & $1.87 * *$ & .061 & NS \\
\hline
\end{tabular}

Table 7: Comparison between cases with hospital stay $<4$ days and cases with hospital stay $\geq 4$ days as regard HCG and estradiol. 


\section{DISCUSSION}

The most frequent medical issue during pregnancy is nausea and vomiting, which is referred to as "morning sickness." Before the 20th week of pregnancy,Approximately $75 \%$ of pregnant women experience vomiting ${ }^{9}$ and nausea.

Although the vast majority of pregnant women adjust to their position, vomiting and nausea can be severe in $10 \%$ of cases, leading to nutritional issues ${ }^{10}$.

To prevent maternal [vitamin B1 (thiamine) and $\mathrm{K}$ deficiency, Wernicke's encephalopathy] and fetal/neonatal [preterm birth, intrauterine growth restriction (IUGR), increased insulin resistance, psychological issues in adulthood] consequences, early detection of HG is critical ${ }^{11}$.

Young age, psychiatric disease, female fetus, nulliparity, twin pregnancy, a previous pregnancy characterized by hyperemesis and hyperthyroidism have all been recognized as risk factors for hyperemesis gravidarum $^{12}$.

The cause of HG is uncertain, though it is assumed to be caused by hormonal, metabolic, mental, and psychosomatic diseases ${ }^{13}$.

Many ideas have been offered, implying its complex characterfor example, excessive levels of betachorionic gonadotropin (-hCG) and estradiol (E2), as well as psychological aspects ${ }^{14}$.

The study's main goal was to seehow elevated concentrations of human chorionic gonadotropin and estradiol in the first trimester increased the intensity of hyper emesis gravidarum.

This prospective study was done in El Hussein Hospital and conducted at 80 pregnant women during the first trimester of their ongoing pregnancy with HG. They were subjected to detailed history, physical examination, ultrasonography, estimate serum HCG, and Serum estradiol, a full blood count, ketones in urine (by dipstick), creatinine, urea ands.electrolytes. An extended hospitalization was described as a stay $\geq 4$ days, as this cut-off value demarcates the top quartilef or period of hospitalization in the study population.

The present study showed that the mean age, weight and GA among study cases was $26.9 \pm 6.1$ years, 67.2 $\pm 6.8 \mathrm{Kg}$ and $10.8 \pm 5.2 \mathrm{Weeks}$ respectively. The median parity among cases was 2 children.

While in Atmaca et al. ${ }^{10}$, the mean age, weight and GA among study cases was $26.54 \pm 4.64$ years, $56.79 \pm 10.25 \mathrm{Kg}$ and $9.18 \pm 1.93$ Weeks respectively.

The results demonstrated that the mean HCG was $197434.8 \pm 143817.9$ with a median of 256127 . HCG was high among $55 \%$ of cases. The mean estradiol was $2141 \pm 946.5$ with a median of 2200 . Estradiol was high among $42.5 \%$ of cases. More than half of cases $(51.25 \%)$ were admitted in hospital for 4 or more days which mean sever hyper emesis gravidarum.

While in Oruç et al. ${ }^{15}$, the mean HCG was 95415.81 \pm 44719.57 and the mean estradiol was $1677.84 \pm$ 982.5 .
In this study,there was no considerable linkbetween personal and medical data on one hand (age, parity, GA, weight and $\mathrm{Hb}$ ) and HCG level and estradiol level on the other hand, with exception of GA where it showed a positive significant correlation with estradiol level.

Our results are supported by another study in which no significantlinkage between physiological characteristics (age and weight) of respondents and HCG level ${ }^{16}$.

From the results of this study, there was no significant correlation between HCG and estradiol level this result against the scientific background for this finding this may be because number of patients was not enough.

The results demonstrated that no significant difference between cases with hospital stay $<4$ days and cases with hospital stay $\geq 4$ days as regard personal and medical data (age, GA, weight and $\mathrm{Hb}$ and parity).

Our findings support those of Atmaca et al. ${ }^{10}$, who found no clinically important variations in age, weight, height, gravidity, parity, or gestational age across the groups.

Unlu et al. ${ }^{17}$ discovered that the maternal age of HG patients was substantially greater than the control group.

There was a significantly considerable impact in this study between patients with hospital stay< 4 days and cases with hospital stay $\geq 4$ days as regard HCG.

The relationship between hCG release and NVP signs is largely responsible for this result,both of which reach their apex during weeks 9 and 12 of pregnancy ${ }^{8}$.

In a review published in 2014, 18 studies found a relationship between elevated hCG levels and NVP or HG,13 studies, on the other hand, revealed no such correlation. $^{18}$

The Generation $\mathrm{R}$ study examined the amount of hCG in 8,195 participants and found a connection between hCG and common NVP signs ${ }^{19}$.

Our results supported by another study where serum level of HCG was significantly associated with severity of $\mathrm{HG}^{20}$.

During a normal pregnancy, the placental tissues are densely invaded by mononuclear phagocytes and lymphocytes. trophoblast is the main origin of IL-6 during pregnancy which controls the secretion of hCG. Excessive activity of granulocytes and high concentration of trophoblast-derived cytokines might be involved in hyperemesis gravidarum. The impact of increased amounts of hCG in brain areas directly implicated in nausea or upper gastrointestinal tract or can rise thyroid hormone and estradiol which has the ability to affect nausea ${ }^{21}$.

Another studydiscovered a relationship between elevated hCG levels and the incidence of HG. The peak of hCG secretion in the first trimester coincides with the time of hyperemesis gravidarum that is generally seen. In women with twin and molar pregnancy which is associated with high HCG levels, 
HG is more common than normal pregnant women 22

This result agrees with what of Derbent et al. ${ }^{23}$ stated that "Hyperemesis gravidarum is associated with elevated levels of HCG, and such changes are independent of serum indicators of thyroid and liver function". This opens field for further studies for predication of hyperemesis gravidarum and its outcome.

Also, this agrees with what Peled et al. ${ }^{24}$ stated in a study investigatedthe effects of serious hyperemesis gravidarum on the triple test (alfafeto-protein, estriol, Human Chorionic Gonadotropin(hCG)) screening results, the study resulted in "An increase in HCG levels in patients with severe hyperemesis gravidarum affects the triple test screen results. When counseling patients about their overall risk of chromosomal abnormalities, this information should be taken into account."

On the contrary,Derbent et al. ${ }^{23}$ concluded that no evidence of a link between high hCG levels and the development of HG. Also, there was no significant correlation between HG risk and HCG values ${ }^{17}$.

There was no proven link between hCG values and HG in a retrospective cohort study of 4,372 pregnant women after in vitro fertilization ${ }^{25}$.

The current study results showed there were no significant difference between cases with hospital stay $<4$ days and cases with hospital stay $\geq 4$ days was present as regard estradiol.

This is in agreement with Tan et al., ${ }^{22}$ who stated that high estradiol level was not associated with prolonged stay or with more severe HG.Women with hyperemesis have increased estrogen levels than women without hyperemesis, according to another study $^{26}$.

The fact that people with HG have high estrogen levels in their blood suggests that estrogen plays a role in the disease's development. Furthermore, the negative effects of estrogen-containing birth control pills include nausea and vomiting. Estrogen lowers intestinal motility and slows gastric emptying, as well as causing fluid shifts that lower stomach acidity and promote H. pylori growth. Pregnant smokers have decreased estrogen levels and are more likely to get $\mathrm{HG}^{27}$.

The fact that women with HG are more likely to suffer nausea while taking contraceptives with a mix of estrogen and progesterone supports a function for the two hormones ${ }^{8}$. Total estradiol and HG studies, like hCG, are contradictory ${ }^{18}$.

Estradiol can leads to nausea in non-pregnant women by disrupting the frequency and direction of stomach contractions. The reason of this disturbance is uncertain, but it is thought to be hormone signaling, which disrupts slow-wave stomach rhythms significantly. The potency of cholecystokinin (CCK) is increased by raising the sensitivity of vagal CCK type A receptors in the gut, which raises the potency of cholecystokinin (CCK). CCK reduces food intake by slowing stomach emptying and activating afferent neurons of the subdiaphragmatic vagal nerve. 28 .

\section{CONCLUSION}

Early-pregnancy hyperemesis gravidarum is a disorder highlighted by extreme vomiting,nausea, and anorexia, leading to dehydration and weight loss. There is a substantial association between HCG and hyperemesis gravidarum but, no significant association between estradiol and hyperemesis gravidarum, there is a substantial association between the presence of acetone and the severity of hyperemesis gravidarum, according to our findings. As a result, the higher the HCG levels, the more severe the hyperemesis gravidarum; ( i.e high levels of HCG increase the severity of HG).

\section{REFERENCES}

1. McCarthy FP, Lutomski JE, Greene RA. Hyperemesis gravidarum: current perspectives. International journal of women's health. 2014; 6: 719.

2. Cunningham $\mathrm{F} G$, Leveno $\mathrm{K}$ J, Bloom $\mathrm{S} \mathrm{L}$. Hyperemesis gravidarum. Williams Obstetrics $22^{\text {nd }}$ ed. 3 ; (49): p 1113-14. Copyright $\odot 2005$, by the McGraw-Hill Companies, Inc, 2005.

3. Deshpande AD, Gazmararian JA, Petersen R, Jamieson DJ, Schild L, Adams MM et al. Hospitalizations during pregnancy among managed care enrollees. ObstetGynecol. 2002; 100:94-100.

4. Best Pract Res ClinGastroenterol Ismail SK, Kenny L. Review on hyper emesis gravidarum. 2007; 21:755-69.

5. Jueckstock JK, Kaestner R, Mylonas I. Managing hyperemesisgravidarum: a multimodal challenge, BMC Medicine. 2010; 8: 46-58.

6. GabraA, Habib H and Gabra M. Hyperemesis Gravidarum, Diagnosis, and Pathogenesis. Crit Care ObstGyne. 2018; 5(1): 1-5.

7. Goodwin TM. Hyperemesis gravidarum. Progress in Obstetrics and Gynecology, 17; 4: 49-64 John Stud, Seang Lin Tan and Frank (Eds). Copyright $\odot 2006$, Elsevier Science limited ISBN. 2006; 0-443-10313-5

8. Bustos M, Venkataramanan R, Caritis S. Nausea and vomiting of pregnancy-What's new? Autonomic Neuroscience. 2017; 202: 62-72.

9. Maken ZH, Gohar A, Rauf A. Effect of Vitamin B6 and Acupressure on Vomiting Symptoms in Pregnant Women with Hyperemesis Gravidarum. American Journal of Biomedical and Life Sciences. 2021; 9(1): 29-35.

10. Atmaca U, Atalay M, Özçimen N. Investigating maternal serum thyroid hormone, beta-human chorionic gonadotropin (beta-HCG) and free betaHCG levels in hyperemesis gravidarum. Erciyes Medical Journal/Erciyes Tip Dergisi. 2015; 37(4).

11. Ioannidou P, Papanikolaou D, Mikos $\mathrm{T}$ et al. Predictive factors of hyperemesis gravidarum: A systematic review. European Journal of Obstetrics \& Gynecology and Reproductive Biology. 2019; 238: $178-87$. 
12. FiaschiL, Nelson-Piercy C, Tata LJ. Hospital admission for hyperemesis gravidarum: a nationwide study of occurrence, reoccurrence and risk factors among 8.2 million pregnancies. Hum Reprod . 2016; 31: 1675-1684

13. Austin K, Wilson K, Saha S. Hyperemesis gravidarum. Nutrition in Clinical Practice. 2019; 34(2): 226-41.

14. Kim HY, Cho GJ, Kim SY et al. Pre-Pregnancy Risk Factors for Severe Hyperemesis Gravidarum: Korean Population Based Cohort Study. Life. 2021; 11(1): 12.

15. Oruç AS, Mert I, Akturk M et al. Ghrelin and motilin levels in hyperemesis gravidarum. Archives of gynecology and obstetrics. 2013; 287(6): 108792.

16. Kasande AJ, Eze ED, Ezekiel I et al. Alteration of Human Chorionic Gonadotropin Levels among Pregnant Women with Morning Sickness Attending Antenatal Care Services at Ishaka Adventist Hospital, Uganda. Journal of Biosciences and Medicines. 2017; 5(8): 55-77.

17. UnluBS, Energin H, Yildiz Y. Maternal serum pregnancy-associated plasma protein-A levels in hyperemesis gravidarum: a prospective case control study. Clinical and experimental obstetrics \& gynecology. 2014; 41(5): 534-536.

18. Niemeijer MN, Grooten IJ, Vos N. Diagnostic markers for hyperemesis gravidarum: a systematic review and metaanalysis. American journal of obstetrics and gynecology. 2014; 211(2): 150-e1.

19. KorevaarTI, Steegers EA, de Rijke YB. Reference ranges and determinants of total hCG levels during pregnancy: the Generation R Study. European journal of epidemiology. 2015; 30(9): 1057-66.

20. Garshasbi A, Ghazanfari T, Zayeri F. Maternal serum levels of interleukin- 6 and $\beta$-hCG in women with hyperemesis gravidarum in the first trimester of pregnancy. Journal of Basic and Clinical Pathophysiology. 2017; 5(1): 13-8.

21. Agmon N, Sade S, Pariente G. Hyperemesis gravidarum and adverse pregnancy outcomes. Archives of gynecology and obstetrics. 2019; 300(2): 347-53.

22. Tan PC, Tan NC, Omar SZ. Effect of high levels of human chorionic gonadotropin and estradiol on the severity of hyperemesis gravidarum. Clinical Chemistry and Laboratory Medicine (CCLM). 2009; 47(2): 165-71.

23. Derbent A, Yanik F, Simavli S. First trimester maternal serum PAPP-A and free beta-HCG levels in hyperemesis gravidarum. Prenatal Diagnosis. $2011 ; 450-3$

24. Peled Y, Melamed N, Krissi H. The impact of severe hyperemesis gravidarum on the triple test screening results. The Journal of Maternal-Fetal \& Neonatal Medicine. 2012; 25(6): 637-8.

25. Dypvik J, Pereira AL, Tanbo TG. Maternal human chorionic gonadotrophin concentrations in very early pregnancy and risk of hyperemesis gravidarum: A retrospective cohort study of 4372 pregnancies after in vitro fertilization. European Journal of Obstetrics \& Gynecology and Reproductive Biology. 2018; 221: 12-6.

26. Lukanova A, Surcel HM, Lundin E. Circulating estrogens and progesterone during primiparous pregnancies and risk of maternal breast cancer. Int $J$ Cancer. 2012; 130: 910-20.

27. Cengiz H, Dagdeviren H, Caypinar. Plasma serotonin levels are elevated in pregnant women with hyperemesis gravidarum. Archives of gynecology and obstetrics. 2015; 291(6): 1271-6.

28. Mauvais-Jarvis F, Clegg DJ, Hevener AL. The role of estrogens in control of energy balance and glucose homeostasis. Endocrine reviews. 2013; 34(3): 309-38. 